\title{
GROUPS WITH PARAMETRIC EXPONENTS
}

\author{
BY \\ ROGER C. LYNDON(')
}

1. Introduction. Elsewhere $\left({ }^{2}\right)$ we consider the problem of characterizing all the solutions $x$, in a free group on generators $a_{1}, a_{2}, \cdots, a_{r}$, of a given equation $w\left(x, a_{1}, a_{2}, \cdots, a_{r}\right)=1$. The totality of solutions can be described as the set of all values assumed by a certain finite set of group theoretic expressions upon substituting integers for certain parameters appearing in these expressions as exponents. As a trivial example, the solutions of $x^{-1} a_{1}^{-1} x a_{1}=1$ are all values assumed by $a_{1}^{\nu}$ as $\nu$ runs through the integers. The general result, and our method of obtaining it, has led us to the study of such expressions, or "words," that contain certain parameters $\nu_{1}, \nu_{2}, \cdots, \nu_{d}$ as exponents. These words may be taken in a natural way as representing elements of a group $G$ that admits additional algebraic operations of raising an element $g$ of $G$ to an exponent $\alpha$, where $\alpha$ is any element of the ring $Z\left[\nu_{1}, \nu_{2}, \cdots, \nu_{d}\right]$ of all polynomials in indeterminates in $\nu_{1}, \nu_{2}, \cdots, \nu_{d}$ with integer coefficients.

More generally, if $X$ is any associative ring with 1 , we call a group $G$ an $X$-group if it is equipped with additional operations $g \rightarrow g \cdot \alpha$ for each $\alpha$ in $X$, subject to the following axioms:

$$
g^{\cdot 1}=g, \quad g^{\cdot(\alpha+\beta)}=g^{\cdot \alpha} g^{\cdot \beta}, \quad g^{\cdot(\alpha \beta)}=\left(g^{\cdot \alpha}\right)^{\cdot \beta}, \quad g(h g)^{\cdot \alpha}=(g h)^{\cdot \alpha} g .
$$

We hasten to note that if $n$ is an integer, the axioms imply $g^{\cdot n}=g^{n}$, so that we may omit all dots. Like the power maps, $g \rightarrow g^{n}$, these operations are not required to define endomorphisms: we do not require that $(g h)^{\alpha}=g^{\alpha} h^{\alpha}$. The last axiom requires that, like the power maps, these operations commute with all inner automorphisms: $\left(g^{-1} h g\right)^{\alpha}=g^{-1} h^{\alpha} g$.

The appropriateness of these axioms to our purpose is demonstrated by the fact, proved below, that for $X=Z\left[\nu_{1}, \nu_{2}, \cdots, \nu_{d}\right]$, a word $\omega$ in the letters $a_{1}, a_{2}, \cdots, a_{r}$ containing parameters $\nu_{1}, \nu_{2}, \cdots, \nu_{d}$ assumes the value 1 in the free group on $a_{1}, a_{2}, \cdots, a_{r}$ under all substitutions for the parameters if and only if it reduces to the "empty word" 1 by virtue of the given axioms.

The main result of this paper is the solution of an extended word problem for free $X$-groups, $X=Z\left[\nu_{1}, \nu_{2}, \cdots, \nu_{d}\right]$. An effective process is exhibited whereby, given a word $\omega$, as above, an element $\alpha$ in $X$ is determined such that, under any substitution of integers for the $\nu_{i}, \omega$ assumes the value 1 if and only if $\alpha$ assumes the value 0 .

Received by the editors December 28, 1959.

(1) This work was begun while the author was visiting at the University of California, Berkeley, in 1956, and has been supported in part by grants from the National Science Foundation.

(2) Equations in free groups, Trans. Amer. Math. Soc. vol. 96 (1960) pp. 445-457. 
Although we study $X$-groups only for $X=Z\left[\nu_{1}, \nu_{2}, \cdots, \nu_{d}\right], d \geqq 0$, and, indeed, only free $X$-groups, we believe the concept is of some more general interest. For $X=Z$, the ring of integers, the concept of $X$-group reduces to that of ordinary group. For $X=Z_{m}$, the integers modulo some $m$, the $X$ groups are just those ordinary groups in which all elements have order dividing $m$. For $X$ the field of rationals, the $X$-groups are just those groups in which "extraction of roots" is always possible and unique; questions related to this have been studied by B. H. Neumann [7], A. I. Malcev [6], R. Baer [1], P. G. Kontorovich [4], and others, in particular, recently by G. Baumslag [2], who gives further references. Baumslag considers more generally the case that $X$ consists of those rationals that can be written with denominator a product of primes belonging to some prescribed set. For $X$ the field of real numbers, the formalism of $X$-groups is at least superficially suggestive of certain considerations in the local theory of Lie groups.

Taking a quite different approach, P. Hall [3] and also M. Lazard [5] have introduced groups admitting exponents from a ring more general than the ring of rational integers, and it is clear that there is some overlap between the groups considered by them and $X$-groups. It may also be noted that $\mathrm{V}$. A. Tartakovski [8] has made use of a limited class of what we call parametric words.

Finally, I want to record that, although the connection is perhaps remote, my interest in the present problem derives from a question of A. Tarski, whether the "elementary theory" of free groups is decidable.

2. Parametric words. The existence of a free $X$-group $F$, for any $X$, on any number of generators $a_{1}, a_{2}, \cdots, a_{r}$ follows from the fact that the axioms for $X$-groups can be written as identical equations. However, confining ourselves henceforth to the case that $X=Z\left[\nu_{1}, \nu_{2}, \cdots, \nu_{d}\right], d \geqq 0$, we shall need a more constructive description of the free $X$-group $F$ in terms of "parametric words" representing its elements.

The elements of the ordinary free group $F_{0}$ on generators $a_{1}, a_{2}, \ldots, a_{r}$ are represented by "words" or "formal products" $\omega=a_{i_{1}}^{\epsilon_{1}} \cdots a_{i_{n}}^{\epsilon_{n}}, n \geqq 0$, $\epsilon_{i}= \pm 1$, of letters $a_{i}^{\epsilon}$. (Rigorously, one may define a word to be a sequence of letters.) A word is reduced if it contains no letter followed by its inverse. Every element of $F_{0}$ is represented by a unique reduced word. The obvious procedure for replacing a given word by a reduced word representing the same group element provides an affirmative solution to the word problem for ordinary free groups.

We shall attempt to parallel all this for the free $X$-group $F$, defining first the concept of "parametric word," then a subset of "normal words," and finally solving for $F$ the generalization stated above of the word problem.

We begin with the observation that the ordinary subgroup $F_{0}$ of $F$ generated by $a_{1}, a_{2}, \cdots, a_{r}$ is in fact free on these generators. To see this, let $G$ be the ordinary free group on a set of generators $b_{1}, b_{2}, \cdots, b_{r}$. If $\rho$ is any 
retraction of $X$ onto $Z$, for example that defined by setting all $\rho \nu_{i}=0$, then we can make $G$ into an $X$-group $G^{\prime}$ by defining $g^{\alpha}=g^{\rho \alpha}$ for all $g$ in $G$ and $\alpha$ in $X$. Since $F$ is the free $X$-group on generators $a_{1}, a_{2}, \cdots, a_{r}$, and $G^{\prime}$ is an $X$-group generated by $b_{1}, b_{2}, \cdots, b_{r}$, there exists an $X$-group homomorphism $\phi$ of $F$ onto $G^{\prime}$ for which $\phi a_{i}=b_{i}, i=1,2, \cdots, r$. But then the restriction of $\phi$ to $F_{0}$ is an ordinary group homomorphism of $F_{0}$ onto $G$, and it is in fact clear that it is an isomorphism, whence it follows that $F_{0}$ is free on the generators $a_{1}, a_{2}, \cdots, a_{r}$. We remark in passing that, taking $F_{0}$ in the role of $G$, every retraction $\rho$ of $X$ onto $Z$ induces a retraction $\bar{\rho}$ of $F$ onto $F_{0}$, under which $\bar{\rho}\left(g^{\alpha}\right)=(\bar{\rho} g)^{(\rho \alpha)}$.

Starting with $F_{0}$, we define an ascending chain of ordinary subgroups of $F$ by letting $F_{t+1}$ be the ordinary subgroup of $F$ generated by all $g^{\alpha}$ where $g$ is in $F_{t}$ and $\alpha$ is in $X$. The union of this chain, as a subset of $F$ containing the generators and closed under all the $X$-group operations, must constitute all of $F$. It follows that each element $w$ of $F$ is contained in some $F_{t}$; if $t>0$, w may be represented by an expression $\omega=\omega^{\alpha_{1}} \omega_{2}^{\alpha_{2}} \cdots \omega_{n}^{\alpha_{n}}$ where the $\omega_{i}$ are expressions representing elements of $F_{t-1}$ and the $\alpha_{i}$ are in $X$. If we take the ordinary words $\omega$ representing elements of $F_{0}$ as words of height 0 , the way is open to define inductively what we mean by a word of height $t$, representing an element $w$ of $F_{t}$. In view of this induction, supposing the word problem solved for $F_{t-1}$, we may more conveniently replace the $\omega_{i}$ by the elements $w_{i}$ in $F_{t-1}$ that they represent, and, tentatively, define a word of height $t$, for $t>0$, to be a "formal product" $\omega=w_{1}^{\alpha_{1}} w_{2}^{\alpha_{2}} \cdots w_{n}^{\alpha_{n}}, n \geqq 0$, where all $w_{i}$ are in $F_{t-1}$, and all $\alpha_{i}$ are in $X$. Rigorously, we may interpret the "formal product" as a sequence of ordered pairs, $\omega=\left(\left(w_{1}, \alpha_{1}\right),\left(w_{2}, \alpha_{2}\right), \cdots,\left(w_{n}, \alpha_{n}\right)\right)$; but in practice no ambiguity will arise from the more suggestive product notation.

The desirability of amplifying this tentative definition of a "word" becomes apparent as soon as we attempt to single out a subclass that is in some reasonable sense "reduced." For these we should like to ensure that, under any retraction $\rho$, there is no cancellation between adjacent factors $\bar{\rho}\left(w_{i}^{\alpha_{i}}\right)$. The example $\omega=a_{1}\left(a_{2} a_{1}\right)^{\alpha}$ shows that we must consider separately retractions $\rho$ according to the sign of $\rho \alpha$. We are led thus to replace $\omega$ by three "words with side conditions": $(\omega, \rho \alpha>0),(\omega, \rho \alpha=0),(\omega, \rho \alpha<0)$. In anticipation of iterating this sort of trichotomy, we take as our formal definition of a word a pair $(\omega, C)$, where $\omega$ is as before and $C$ is any finite set of formal expressions of the type $\rho \alpha<\rho \beta$ or $\rho \alpha=\rho \beta$, for $\alpha$ and $\beta$ elements of $X$.

It is clear what it means for a given retraction to satisfy a given set of condition $C$. The values of a word $(\omega, C)$, where $\omega$ represents the element $w$ of $F$, are all elements $\bar{\rho} w$ in $F_{0}$ for $\rho$ satisfying $C$. The values of a set of words are those of its members. Our aim is to define a class of "normal words," and to show that any given word effectively determines a finite set of normal words which is equivalent to it in the sense of having exactly the same set of values. But we shall use a more constructive definition of equivalence. Two 
sets of words will be called equivalent if it is possible to pass from one to the other by a succession of steps of the following three kinds.

(E1) replace $\left(\omega, C_{1}\right), \cdots,\left(\omega, C_{h}\right)$ by $\left(\omega, D_{1}\right), \cdots,\left(\omega, D_{k}\right)$ where exactly the same retractions satisfy one of the $C_{i}$ as satisfy one of the $D_{j}$;

(E2) replace $(\omega, C)$ by $\left(\omega^{\prime}, C\right)$ where $C$ contains the condition $\rho \alpha=\rho \beta$ for some $\alpha$ and $\beta$ in $X$, and $\omega$ and $\omega^{\prime}$ differ only in that one contains $\alpha$ at certain places where the other contains $\beta$;

(E3) replace $(\omega, C)$ by $\left(\omega^{\prime}, C\right)$ where $\omega=\omega^{\prime}$ follows from the axioms for $X$ groups.

We note that (E1) with $h=1$ and $k=0$ permits us to delete a word $(\omega, C)$ in case $C$ is inconsistent, that is, is satisfied by no retraction $\rho$. Likewise the case $h=1$ and $k=1$ permits us to replace $C$ by any equivalent set of conditions, with the result that we need not pay close attention to the precise manner of formulation of the conditions $C$.

We define a normal word of height 0 to be any pair $(\omega, C)$ where $\omega$ is an ordinary reduced word representing an element of $F_{0}$, and $C$ any consistent set of conditions. The definition of a normal word of height $t$, for $t>0$, requires a number of clauses, which we now formulate.

In a normal word $(\omega, C)$, where $\omega=w_{1}^{\alpha_{1}} \cdots w_{n}^{\alpha_{n}}$, we shall require that $n \geqq 1$, and that $\alpha_{i}=1$ for odd $i$. Changing notation, we can write

$$
\omega=u_{1} v_{1}^{\alpha_{1}} u_{2} v_{2}^{\alpha_{2}} \cdots u_{m} v_{m}^{\alpha_{m}} u_{m+1}
$$

where all $u_{i}$ and $v_{i}$ are in $F_{t-1}$, all $\alpha_{i}$ are in $X$, and $m \geqq 0$.

Our first condition is the following:

(N0) each $u_{i}$ is represented by some $\xi_{i}$, and each $v_{i}$ by some $\eta_{i}$, such that all the $\left(\xi_{i}, C\right)$ and $\left(\eta_{i}, C\right)$ are normal words of height $t-1$.

The next condition enforces a certain measure of uniqueness on the parts $v_{i}^{\alpha_{i}}$. Before stating it, we define a word $(\eta, C)$ to be primitive if it is not equivalent to any word $\left(\zeta^{\beta}, C\right)$ where $\beta \neq \pm 1$. We now state the next condition:

(N1) $C$ contains all the conditions $\rho \alpha_{i}>0$, and $C$ does not imply a condition $\rho \alpha_{i}<k$ for any $i=1,2, \cdots, m$, and $k$ in $Z$; each $\left(\eta_{i}, C\right)$ is primitive, and, if $t \geqq 2$, is not equivalent to any word $(\zeta, C)$ of height $t-2$.

In particular, the latter part of Condition N1 will ensure that no $v_{i}^{\alpha_{1}}$ can be absorbed into adjoining $u_{i}$ or $u_{i+1}$.

Our next condition will turn out to be an immediate consequence of Condition N0; but, since we are deferring all proofs to later sections, we state it here separately.

(N2) For each $v_{i}$ there exist two letters $L\left(v_{i}\right)$ and $R\left(v_{i}\right)$ such that, for all $\rho$ satisfying $C$, the element $\bar{\rho} v_{i}$ in $F_{0}$ is represented by a nontrivial reduced word beginning with $L\left(v_{i}\right)$ as left letter and ending with $R\left(v_{i}\right)$ as right letter; and each $u_{i} \neq 1$ must satisfy the analogous condition.

A product $a_{1} a_{2} \cdots a_{n}$ will be called reduced (relative to $C$ ) if each $a_{i} \neq 1$ 
possesses letters $L\left(a_{i}\right)$ and $R\left(a_{i}\right)$ in the manner of Condition N2, and if, for $1 \leqq i \leqq j \leqq n, a_{i+1}=a_{i+2}=\cdots=a_{j-1}=1$ implies $R\left(a_{i}\right) L\left(a_{j}\right) \neq 1$; in short, in the product there is no cancellation. We shall of ten insert a dot, writing $a \cdot b$, to express that the product $a b$ is reduced relative to $C$. In particular, writing $a \cdot a$ expresses that $a$ is cyclically reduced, that is, it does not begin and end in letters that are inverse to each other. With this we can state the condition that excludes the possibility of cancellation between factors in a normal word:

(N3) $u_{1} v_{1} v_{1} u_{2} v_{2} v_{2} \cdots u_{m} v_{m} v_{m} u_{m+1}$ is reduced relative to $C$.

There remains some ambiguity in the normal form as stipulated thus far in that a part of one factor may be transferred to an adjacent factor. The equation $a_{1}\left(a_{2} a_{1}\right)^{\alpha}=\left(a_{1} a_{2}\right)^{\alpha} a_{1}$, which is an instance of one of the axioms, shows that we can not hope to resolve this ambiguity in a symmetric manner. We choose to "shift" the parts $v_{i}^{\alpha_{i}}$ as far to the left as possible:

(N4a) if $u_{i} \neq 1,1 \leqq i \leqq m, R\left(u_{i}\right) \neq R\left(v_{i}\right)$;

(N4b) if $u_{i}=1,1 \leqq i \leqq m, R\left(v_{i}\right) \neq R\left(v_{i+1}\right)$.

Condition $\mathrm{N} 4$ is compatible with a weakened form of the symmetric counterpart of $\mathrm{N} 4 \mathrm{a}$, requiring that no $u_{i+1}$ begin with the whole of preceding $v_{i}$ :

(N5) for $1 \leqq i \leqq m,\left(\xi_{i+1}, C\right)$ is not equivalent to any $\left(\eta_{i} \zeta, C\right)$ where $\zeta$ represents an element $z$ of $F_{t-1}$, and $u_{i+1}=v_{i} \cdot z$, reduced relative to $C$.

We note that the corresponding weakened counterpart of $N 4 \mathrm{~b}$, requiring that, if $u_{i}=1$, not $v_{i+1}=v_{i} \cdot z$, is incompatible with the remaining conditions; for this would lead to the following vicious circle of "reductions":

$$
x^{\alpha}(x y)^{\beta} \rightarrow x^{\alpha+1} y(x y)^{\beta-1} \rightarrow x^{\alpha+1}(y x)^{\beta-1} y \rightarrow x^{\alpha}(x y)^{\beta-1} x y \rightarrow x^{\alpha}(x y)^{\beta} .
$$

A word of height $t$, for $t>0$, will be called normal if it satisfied all the conditions N0, N1, N2, N3, N4, N5 set forth above.

\section{Reduction to normal form.}

THEOREM I. There exists an effective procedure for associating with each word an equivalent finite set of normal words.

The theorem will be proved by induction on the height of the given word. For words of height 0 the assertion is obvious. Let $t>0$ and assume

Proposition 1. If $(\omega, C)$ is a word of height $t-1$, then $(\omega, C)$ is equivalent to a set of normal words $\left(\omega_{1}, C_{1}\right), \cdots,\left(\omega_{n}, C_{n}\right)$.

From this we must derive the corresponding assertion, Proposition $1^{\prime}$, for words of height $t$. In fact, we shall take as our induction hypothesis not only Proposition 1 but also certain auxiliary Propositions $2,3, \cdots, 7$, concerning words of height $t-1$, from which we must derive not only Proposition $1^{\prime}$, but also the corresponding Propositions $2^{\prime}, 3^{\prime}, \cdots, 7^{\prime}$. All of these propositions are obvious for $t=0$. 
In this section we derive Proposition $1^{\prime}$ from $1,2, \cdots, 7$, and in the next section derive $2^{\prime}, 3^{\prime}, \cdots, 7^{\prime}$ from the same hypothesis. It will be evident from inspection that the reduction process is effective.

At various stages of the reduction process that will be described below, we shall have occasion to replace a word $(\omega, C)$ by three words $\left(\omega, C_{1}\right),\left(\omega, C_{2}\right)$, $\left(\omega, C_{3}\right)$ obtained by adjoining to $C$ each of the conditions $\rho \alpha<0, \rho \alpha=0, \rho \alpha>0$. The argument from this point on will deal separately with each of these three words. To simplify notation we shall drop subscripts and instead of saying that we are in case $C_{1}, C_{2}$ or $C_{3}$, we shall say rather that $C$ contains $\rho \alpha<0$, $\rho \alpha=0$ or $\rho \alpha>0$. Clearly this is permissible provided we can effect the reduction of $(\omega, C)$ to some normal $\left(\omega^{\prime}, C\right)$ by making, at a finite number of junctures well determined by $\omega$, for $\alpha$ determined by $\omega$, the assumption that $C$ contains one or another of $\rho \alpha<0, \rho \alpha=0$ and $\rho \alpha>0$.

This convention, whereby we regard $C$ as unchanged throughout the argument, enables us to suppress mention of $C$. In this spirit we shall speak of the word $\omega$ instead of the word $(\omega, C)$. We shall write $\alpha<0, \alpha=0, \alpha>0$ to express that $\rho \alpha<0, \rho \alpha=0, \rho \alpha>0$ belongs to $C$. We shall speak of all $\rho$, rather than of all $\rho$ that satisfy $C$. It is worth emphasizing that we must not suppose that $C$ contains one out of an infinite set of alternatives. Thus, although all $\rho \alpha=k$ for some $k$ in $Z$, we can not suppose that $C$ necessarily contains one of the inequalities $\rho \alpha \leqq k$. If $C$ does contain such an inequality, we write $\alpha \in Z$, and we write $\alpha \notin Z$ to express that $C$ contains no such inequality.

We now state those propositions that make up the rest of the induction hypothesis.

Proposition 2. If $(\omega, C)$ is a word of height $t-1$, representing $w \neq 1$ in $F$, and is not equivalent to $(1, C)$, where 1 here denotes the "empty" word, then there exist letters $L(\omega, C)$ and $R(\omega, C)$ such that, for all $\rho$ satisfying $C$, the reduced word representing $\bar{\rho} w$ in $F_{0}$ begins with $L(\omega, C)$ as its left letter and ends with $R(\omega, C)$ as its right letter.

Apart from our convention regarding $C$, we should have to say rather that arbitrary $(\omega, C)$ is effectively equivalent to a finite set of words $\left(\omega_{i}, C_{i}\right)$, each with the property asserted by the proposition. In view of our convention, we can simplify the statement of the proposition by suppressing all reference to $C$. In view of the fact that we are assuming Proposition 1 we can go further: not only does every $\omega$ of height $t-1$ represent an element $w$ of $F_{t-1}$, but every $w$ in $F_{t-1}$ is represented by normal $(\omega, C)$ for some $\omega$. (Literally, $C$ has been chosen so that this is true for every $w$ that comes up for our consideration.) Thus we can as well speak of all $w$ as of all $\omega$, and restate the proposition more perspicuously.

Proposition 2 (restated). If $w$ is in $F_{t-1}$ and $w \neq 1$, then there exist letters $L(w)$ and $R(w)$ such that all $\bar{\rho} w$ begin with $L(w)$ and end with $R(w)$. 
(The fact that $w=1$ implies $\omega=1$, as well as conversely, follows from the original statement of the proposition, since 1 has no first or last letter.)

Proposition 3. If $a$ is in $F_{t-1}$ there exist $b$ and $c$ in $F_{t-1}$ such that $a=b^{-1} \cdot c \cdot b$ reduced, and $c$ is cyclically reduced (that is, $c^{2}=c \cdot c$ ).

Proposition 4. If $a$ is in $F_{t-1}$, then there exist $b$ in $F_{t-1}$ and $\alpha$ in $X$ such that $a=b^{\alpha}$, and $b$ is primitive (that is, $b=c^{\beta}$ implies $\beta= \pm 1$ ).

Proposition 5. If $a$ and $b$ are in $F_{t-1}, b$ cyclically reduced, primitive, and not in $F_{t-2}$, then there exist $a_{1}, b_{1}, b_{2}$ in $F_{t-1}$ and $k$ in $Z$ such that $b=b_{1} \cdot b_{2}$, $a=a_{1} \cdot b_{2} b^{k}$ reduced, $k \geqq 0, b_{1} \neq 1$, and, if $a_{1} \neq 1, R\left(a_{1}\right) \neq R\left(b_{1}\right)$.

Proposition 6. If $a$ and $b$ are in $F_{t-1}, a \neq b$, both are cyclically reduced, primitive, and not in $F_{t-2}$, then there exist $a_{1}, a_{2}, b_{1}, b_{2}$ in $F_{t-1}$ and $h, k$ in $Z$ such that

$$
\begin{aligned}
& a=a_{1} \cdot a_{2}, \quad b=b_{1} \cdot b_{2}, \quad a_{2} a^{h}=b_{2} b^{k}, \quad h \geqq 0, k \geqq 0, \\
& a_{1} \neq 1, \quad b_{1} \neq 1, \quad \text { and } \quad R\left(a_{1}\right) \neq R\left(b_{1}\right) .
\end{aligned}
$$

Proposition 7. If $a$ and $b$ are in $F_{t-1}$ and $a b=b a$, then there exist $c$ in $F_{t-1}$ and $\alpha, \beta$ in $X$ such that $a=c^{\alpha}, b=c^{\beta}$.

To begin the proof of Proposition 1', we suppose given a word $(\omega, C)$ of height $t$, and must replace it by an equivalent normal word $\left(\omega^{\prime}, C\right)$. That is, we are given a product representation

$$
w=w_{1}^{\beta_{1}} w_{2}^{\beta_{2}} \cdots w_{n}^{\beta_{n}}, \quad n \geqq 0, w_{i} \in F_{t-1}, \beta_{i} \in X,
$$

for an element $w$ of $F_{t}$, and, by means of the axioms, must transform this into a representation satisfying the conditions $\mathrm{N} 0,1,2,3,4,5$ for normality.

Condition N0 follows immediately from Proposition 1 and our convention on $C$. For this proposition ensures that each $w_{i}$ is represented by a finite set of normal $\left(\omega_{j}, C_{j}\right)$ equivalent to $\left(\omega_{i}, C\right)$, and under our convention we suppose $C$ is already one of the $C_{j}$, so that $w_{i}$ is represented by a normal word $\left(\omega_{i}, C\right)$.

To establish Condition N1, we first delete any $w_{i}^{\beta_{i}}=1$. By Proposition 3 we may replace each part $w_{i}^{\beta_{i}}$ by $z_{i} v_{i}^{\beta_{i}} z_{i}^{-1}$, where $v_{i}$ is cyclically reduced, and, by Proposition 4 , after changing notation, we may suppose $v_{i}$ primitive. We now have a product of factors $u$ in $F_{t-1}$ and factors $v^{\alpha}$, which we may suppose are not in $F_{t-1}$, where the $v$ are cyclically reduced and primitive. Replacing any succession of factors $u_{1}, u_{2}, \cdots, u_{k}$ all in $F_{t-1}$ by a single factor $u=u_{1} u_{2} \cdots u_{k}$ in $F_{t-1}$, and inserting factors 1 if necessary, we have

$$
w=u_{1} v_{1}^{\alpha_{1}} u_{2} v_{2}^{\alpha_{2}} \cdots u_{m} v_{m}^{\alpha_{m}} u_{m+1}
$$

satisfying all the clauses of Condition N1 except that possibly some $\alpha_{i}<0$. This is simply remedied by replacing $v_{i}^{\alpha_{i}}$ by $v_{i}^{\prime \alpha_{i}^{\prime}}$ where $v_{i}^{\prime}=v^{-1}$ and $\alpha_{i}^{\prime}=-\alpha_{i}$. 
Condition N2 follows immediately from N0 together with Proposition 2.

To establish Condition N4, we again proceed by induction on $i$, assuming $R\left(v_{j-1} u_{j}\right) \neq R\left(v_{j}\right)$ for all $j<i$ (and, if $i>0, u_{1} \neq 1$, that $R\left(u_{1}\right) \neq R\left(v_{1}\right)$. For $i=0$ this is trivial, and for $i=m$ we are done. If the hypothesis holds for $i$ and fails for $i+1$ there are two cases.

CASE 1. $u_{i} \neq 1$. By Proposition 5 we can write $v_{i}=b_{1} \cdot b_{2}, u_{i}=a_{1} b_{2} v_{i}^{k}$ reduced, $k \in Z, k \geqq 0, b_{1} \neq 1$, and, if $a_{1} \neq 1, R\left(a_{1}\right) \neq R\left(b_{1}\right)$. Then

$$
\begin{aligned}
u_{i} v_{i}^{\alpha_{i}} u_{i+1} & =a_{1} b_{2}\left(b_{1} b_{2}\right)^{\alpha_{i}+k} u_{i+1} \\
& =a_{1}\left(b_{2} b_{1}\right)^{\alpha_{i}+k} b_{1} u_{i+1} \\
& =u_{i}^{\prime} v_{i}^{\prime \alpha_{i}^{\prime}} u_{i+1}^{\prime}
\end{aligned}
$$

where $u_{i}^{\prime}=a_{1}, v_{i}^{\prime}=b_{2} b_{1}, u_{i+1}^{\prime}=b_{1} u_{i+1}, \alpha_{i}^{\prime}=\alpha_{i}+k$. Clearly Conditions N0, 1, 2, 3 are preserved, as well as the hypothesis for $j<i$. Moreover, if $u_{i}^{\prime}=a_{i} \neq 1$, the hypothesis holds for $j=i$, since then $R\left(v_{i-1} u_{i}^{\prime}\right)=R\left(a_{1}\right) \neq R\left(b_{1}\right)=R\left(v_{i}^{\prime}\right)$. If $u_{i}^{\prime}=1$, we are reduced to Case 2 , below.

CASE 2. $u_{i}=1$. By Proposition 6 we can write $v_{i-1}=a_{1} \cdot a_{2}, v_{i}=b_{1} \cdot b_{2}$, $a_{2} v_{i-1}^{h}=b_{2} v_{i}^{k}, h, k \in Z, h, k \geqq 0, a_{1}, a_{2} \neq 1, R\left(a_{1}\right) \neq R\left(b_{1}\right)$. Then

$$
\begin{aligned}
v_{i-1}^{\alpha_{i}-1} v_{i}^{\alpha_{i}} u_{i+1} & =v_{i-1}^{\alpha_{i-1}-h-1} a_{1} b_{2}\left(b_{1} b_{2}\right)^{\alpha_{i}+k} u_{i+1} \\
& =v_{i-1}^{\alpha_{i-1}-h-1} a_{1}\left(b_{2} b_{1}\right)^{\alpha_{i}+k} b_{2} u_{i+1} \\
& =v_{i-1}^{\alpha_{i}^{\prime}-1} u_{i}^{\prime} v_{i}^{\prime \alpha_{i}^{\prime}} u_{i+1}^{\prime}
\end{aligned}
$$

where $u_{i}^{\prime}=a_{1}, v_{i}^{\prime}=b_{2} b_{1}, u_{i+1}^{\prime}=b_{2} u_{i+1}, \alpha_{i-1}^{\prime}=\alpha_{i-1}-h-1, \alpha_{i}^{\prime}=\alpha_{i}+k$. Clearly No, 1, 2, 3 are preserved, and the condition on $j<i$. For $j=i$, we verify that $R\left(v_{i-1} u_{i}^{\prime}\right)=R\left(a_{1}\right) \neq R\left(b_{1}\right)=R\left(v_{i}^{\prime}\right)$. This completes the induction on $i$.

To establish Condition N5, by the symmetric counterpart of Proposition 5 we can write $v_{i}=b_{2} \cdot b_{1}, u_{i+1}=v_{i}^{k} b_{2} a_{1}$ reduced, $k \in Z, k \geqq 0, b_{1} \neq 1$, and, if $a_{1} \neq 1$, $L\left(a_{1}\right) \neq L\left(b_{1}\right)$. Then $v_{i}^{\alpha_{i}} u_{i+1}=v_{i}^{\alpha_{i}+k} b_{1} a_{1}=v_{i}^{\alpha_{i}^{\prime}} u_{i+1}^{\prime}$ where $\alpha_{i}^{\prime}=\alpha_{i}+k, u_{i+1}^{\prime}=b_{1} a_{1}$. Clearly none of Conditions N0,1, 2, 3, 4 are lost. It remains to show that $u_{i+1}^{\prime}=v_{i} \cdot w, w$ in $F_{t-1}$, is impossible. But this gives $b_{1} \cdot a_{1}=b_{2} \cdot b_{1} \cdot w$ reduced, whence $a_{1}=b_{1} \cdot w$, which contradicts the conditions that $b_{1} \neq 1$ and that, if $a_{1} \neq 1, L\left(a_{1}\right) \neq L\left(b_{1}\right)$.

This completes the proof of Proposition 1' from Propositions 1, 2, $\cdots, 6$ (and 7).

4. Proof of the auxiliary propositions. We continue to argue on the basis of Propositions 1 through 7 , together with $1^{\prime}$, which was established in the preceding section.

To prove Proposition $2^{\prime}$, let $w$ in $F_{t}, w \neq 1$. By Proposition $1^{\prime}$ we can suppose $w=u_{1} v_{1}^{\alpha_{1}} \cdots u_{m} v_{m}^{\alpha_{m}} u_{m+1}$, normal. Then, for any $\rho, \bar{\rho} w=\bar{\rho} u_{1}\left(\bar{\rho} v_{1}\right)^{\rho \alpha_{1}} \cdots$ $\left(\bar{\rho} v_{m}\right)^{\rho \alpha_{m}} \bar{\rho} u_{m+1}$, where, by $\mathrm{N} 2,3$, this product is reduced. It follows that if 
$u_{1} \neq 1, \bar{\rho} w$ begins with $L\left(u_{1}\right)$, and with $L\left(v_{1}\right)$ otherwise; similarly $\bar{\rho} w$ ends with $R\left(u_{m+1}\right)$ if $u_{m+1} \neq 1$, and with $R\left(v_{m}\right)$ otherwise.

Corollary. If $a=a_{1} \cdot a_{2} \cdots a_{n}$ reduced, all $a_{i}$ are in $F_{t}$, and not all $a_{i}=1$, then $a \neq 1$.

To prove this, after deleting any $a_{i}=1$, we reason as before that for all $\rho, \bar{\rho} a=\bar{\rho} a_{i} \cdots \bar{\rho} a_{n}$, whence $L(a)=L\left(a_{1}\right), R(a)=R\left(a_{1}\right)$, and consequently $a \neq 1$.

We state now a lemma whose proof is contained in the algorithm given above for establishing Condition N3.

Lemma A. If $a$ and $b$ are in $F_{t}$, then there exist $a_{1}, c$, and $b_{2}$ in $F_{t}$ such that $a=a_{1} \cdot c, b=c^{-1} \cdot b_{2}$, and $a b=a_{1} \cdot b_{2}$.

An important consequence follows.

Lemma B. If $a, b, c$ and $d$ are in $F_{t}$ and $a \cdot b=c \cdot d$, then there exists $e$ in $F_{t}$ such that either

or

$$
a=c \cdot e \text { and } d=e \cdot b \text {, }
$$

$$
c=a \cdot e \quad \text { and } \quad b=e \cdot d .
$$

Proof of Lemma B. By Lemma A, we can write $a=e \cdot a_{2}, c=e \cdot c_{2}, a^{-1} c$ $=a_{2}^{-1} \cdot c_{2}$. If $a_{2} \neq 1, c_{2} \neq 1$, then $b^{-1} a^{-1} c d=b^{-1} a_{2}^{-1} c_{2} d$ is reduced and, by Proposition $2^{\prime}$ (since it clearly has left and right letters), $b^{-1} a^{-1} c d \neq 1$, contrary to hypothesis. By symmetry, we may suppose that $a_{2}=1$. Then $a=e, c=a \cdot c_{2}$, and $a b=c d=a c_{2} d$ implies that $b=c_{2} \cdot e$.

To prove Proposition $3^{\prime}$, let $a \in F_{t}$. By Lemma A, we may write $a=a_{1} \cdot c$, $a=c^{-1} \cdot a_{2}, a^{2}=a_{1} \cdot a_{2}$. Lemma B applied to the relation $a_{1} \cdot c=c^{-1} \cdot a_{2}$ presents two alternatives. First, suppose $a_{1}=c^{-1} \cdot e, a_{2}=e \cdot c$ then $a=c^{-1} \cdot e \cdot c$ where $a^{2}=a^{-1} \cdot e \cdot e \cdot c$, whence $e \cdot e$ and $e$ is cyclically reduced, as required. Otherwise, $c^{-1}=a_{1} \cdot e, c=e \cdot a_{2}$. Then Lemma B applied to $c=e^{-1} \cdot a_{1}^{-1}, c=e \cdot a_{2}$ gives, after replacing $e$ by $e^{-1}$ if necessary, $e=e^{-1} \cdot f$, and substituting, $e=f^{-1} \cdot e \cdot f$. Applying $\rho$ to this equation gives $\bar{\rho} f=1$, and then to the equation $e=e^{-1} \cdot f$ gives $\bar{\rho} e=\bar{\rho} e^{-1}$ whence $\bar{\rho} e=1, e=1$ and $a=1$, cyclically reduced.

LeMma C. If $a=u v^{\alpha}, b=w z^{\beta}$, normal, in $F_{t}$, then either $u=w$ and $v=z$ or else there exist $c$ in $F_{t-1}$ and $h, k \in Z, h, k \geqq 0$, such that $a^{-1} b=v^{-(\alpha-h)} \cdot c \cdot z^{\beta-k}$ reduced.

Proof. By Lemma A we can write $u=e \cdot u_{2}, w=e \cdot w_{2}, u^{-1} w=u_{2}^{-1} \cdot w_{2}$. If $u_{2} \neq 1, w_{2} \neq 1$, then $a^{-1} b=v^{-\alpha} \cdot u^{-1} \cdot w_{2} \cdot z^{\beta}$ reduced and we are done. By symmetry, it suffices to consider the case that $u_{2}=1$, where $a^{-1} b=v^{-\alpha} \cdot u_{2}^{-1} \cdot w_{2} \cdot z^{\beta}$. By Proposition 5, we can write $v=v_{1} \cdot v_{2}, w_{2}=v^{k} v_{1} w_{3}$ reduced, where $v_{2} \neq 1$ and, if $w_{3} \neq 1, L\left(w_{3}\right) \neq L\left(v_{2}\right)$. Then $a^{-1} b=v^{-\alpha} w_{2} z^{\beta}=v^{-(\alpha-k-1)} v_{2}{ }^{-1} w_{3} z^{\beta}$, reduced provided $w_{3} \neq 1$. Henceforth suppose $w_{3}=1$, whence $a^{-1} b=v_{2}^{-1}\left(v_{2} v_{1}\right)^{\alpha-k-1} z^{\beta}$. If $z \neq v_{2} v_{1}$, Proposition 6 yields $a^{-1} b=v_{2}^{-1}\left(v_{2} v_{1}\right)^{-\alpha} c=z^{\beta}=v^{-\alpha^{\prime}} v_{2}^{-1} c z^{\beta^{\prime}}$ reduced, for some $c$ in $F_{t-1}$ and $\alpha^{\prime}=\alpha-h^{\prime}, \beta^{\prime}=\beta-k^{\prime}$. Finally, suppose also that $z=v_{2} v_{1}$. Since $w_{3}=1$, $w=u v^{k} v_{1}$, while by Condition N4 for the normality of $w z^{\beta}$, 
$R(w) \neq R\left(v_{2} v_{1}\right)$. We conclude first that $v_{1}=1$, whence $z=v_{1}=v$, and second that $k=0$, whence $w=u$.

Lemma. D (Uniqueness of normal form). If $a=u_{1} v_{1}^{\alpha_{1}} \cdots v_{m}^{\alpha_{m}} u_{m+1}$, $b=w_{1} z_{1}^{\beta_{1}} \ldots z_{n}^{\beta_{n}} w_{n+1}$, both normal and in $F_{t}$, then $a=b$ implies that $m=n$ and that $u_{i}=w_{i}, v_{i}=z_{i}$, and $\alpha_{i}=\beta_{i}$ for all $i=1,2, \cdots, m$.

Proof. Induction on $m+n$. If $m=n=0$, then $a=u_{1}, b=w_{1}$, and the conclusion is trivial. We also consider separately the case that only one of $m, n$ is 0 , say $m=0$ and $n>0$. In this case $u_{1}^{-1} w_{1} z_{1}^{\beta_{1}}$ has, using Proposition 5, a reduced form $u^{\prime} z_{1}^{\beta^{\prime}}$ for some $u^{\prime}$ and $\beta^{\prime}$, hence $a^{-1} b$ has a reduced form different from 1 , and, by the corollary, $a^{-1} b \neq 1$. For the induction argument, suppose $m, n>0$. Unless $u_{1}=w_{1}$ and $v_{1}=z_{1}$, Lemma $C$ provides for $a^{-1} b$ a nontrivial reduced form, whence $a^{-1} b \neq 1$. Suppose then that $u_{1}=w_{1}, v_{1}=z_{1}$. If also $\alpha_{1}=\beta_{1}$ the conclusion follows by the induction hypothesis applied to $a^{\prime}$ $=u_{2} v_{2}^{\alpha_{2}} \cdots u_{m+1}$ and $b^{\prime}=w_{2} z_{2}^{\beta_{2}} \cdots w_{n+1}$. In the remaining case we may suppose by symmetry that $\gamma=\alpha_{1}-\beta_{1}>0$, whence, after cancelling, we have $a^{\prime}=v_{1}^{\gamma} u_{2} v_{2}^{\alpha_{2}} \cdots u_{m+1}$ equal to $b^{\prime}$. If $\gamma \notin Z$, the product for $a^{\prime}$ is normal, and the induction hypothesis gives $1=w_{2}$, and $v_{1}=z_{2}$, which together with $v_{1}=z_{1}$, contradicts $\mathrm{N} 4$ for $b$. If $\gamma \in Z$, the product for $a^{\prime}$ becomes reduced when we replace $v_{1}^{\gamma} u_{2} z_{1}^{\beta_{2}}$ by a reduced product $u_{2}^{\prime} z_{1}^{\beta^{\prime}}$, and again the induction hypothesis gives $w_{2}=u_{2}^{\prime}=v_{1}^{\gamma} u_{2}, \gamma>0$, which, together with $z_{1}=v_{1}$, contradicts N5 for $b$.

Define $a^{\prime}$ to be a cyclic conjugate of $a$ if, for some $b$ and $c, a=b \cdot c$ and $a^{\prime}=c b$. This relation is transitive, although not symmetric. For suppose $a=b \cdot c, a^{\prime}=c b=d \cdot e$, and $a^{\prime \prime}=e d$. By Lemma A, $c=c^{\prime} \cdot f, b=f^{-1} \cdot b^{\prime}$, and $a^{\prime}=c^{\prime} \cdot b^{\prime}=d \cdot e$. By Lemma $\mathrm{B}$, and symmetry, we may suppose that $c^{\prime}=d \cdot g$, $e=g \cdot b^{\prime}$. Then $a=f^{-1} \cdot b^{\prime} \cdot d \cdot g \cdot f$ reduced, while $a^{\prime \prime}=g b^{\prime} d=(g f)\left(f^{-1} b^{\prime} d\right)$.

Lemma E. Let a, in $F_{t}$, not have any cyclic conjugate a power of an element of $F_{t-1}$. Then there exist integers $t \geqq 0$ and $m>0$, and, for each positive integer $i$, elements $u_{i}, v_{i}$ in $F_{t-1}$ and an element $\alpha_{i}$ in $X$ such that, writing $a_{i}=u_{i} v_{i}^{\alpha_{i}}$,

(1) for all $h \geqq k+2$, the normal form of $a^{h}$ begins

$$
a^{h}=a_{1} a_{2} \cdots a_{k} \cdots ;
$$

(2) for all $i>t, a_{i+m}=a_{i}$;

(3) for $s \geqq t$,

$$
\left(a_{1} a_{2} \cdots a_{s}\right)^{-1} a\left(a_{1} a_{2} \cdots a_{s}\right)=a_{s+1} a_{s+2} \cdots a_{s+m} \text {, normal. }
$$

Proof. For $a$ in $F_{t}$, define $l(a)=n$, the "length" of its normal form $a=w_{1} z_{1}^{\beta_{1}} \cdots w_{n+1}$. For $l(a) \geqq 1$, define $a^{\prime}=\left(w_{1} z_{1}^{\beta_{1}}\right)^{-1} a\left(w_{1} z_{1}^{\beta_{1}}\right)$. If no cyclic conjugate of $a$ is in $F_{t-1}$, evidently $l(a) \geqq l\left(a^{\prime}\right) \geqq 1$.

CASE 1. $l(a)=1$. It will suffice to prove the assertion of the lemma for any cyclic conjugate $u^{-1} a u$ of $a$, where $u$ is in $F_{t-1}$. Replacing $a=w_{1} z_{1}^{\beta_{1}} w_{2}$, normal, by the normal form of $z_{1}^{\beta_{1}} w_{2} w_{1}$ we can suppose $w_{1}=1$. If $w_{2}=w b_{1}^{-1} z_{1}^{-k}$ 
where $z_{1}=b_{1} b_{2}$, in accordance with Proposition 5 , conjugating by $b_{1}^{-1} z_{1}^{-k}$ we can suppose that $a$ is cyclically reduced. If $w_{2}=w b_{2} z_{1}^{k}$ in accordance with Proposition 5, conjugating by $z_{1}^{k}$ we can suppose that $k=0$. Thus we may assume $a=\left(b_{1} b_{2}\right)^{\beta} w b_{2}$, normal and cyclically reduced, where $b_{1} \neq 1$ and, if $w \neq 1, R(w) \neq R\left(b_{1}\right)$.

If $w \neq 1$, conditions (1) and (2) are realized with

$$
a^{h}=\left(b_{1} b_{2}\right)^{\beta} w\left(b_{2} b_{1}\right)^{\beta} b_{2} w\left(b_{2} b_{1}\right)^{\beta} b_{2} w\left(b_{2} b_{1}\right)^{\beta} \ldots .
$$

Suppose $w=1$. Since $a=\left(b_{1} b_{2}\right)^{\beta} b_{2}$ is not a power of the element $b_{1} b_{2}$ in $F_{t-1}$, we conclude that $b_{2} \neq 1$ as well as $b_{1} \neq 1$. We show next that $b_{1} b_{2} \neq b_{2} b_{1}$. For $b_{1} b_{2}=b_{2} b_{1}$ would imply that both $b_{1}$ and $b_{2}$ commuted with the primitive element $z=b_{1} b_{2}$, hence $b_{1}=z_{1}^{\gamma}, b_{2}=z_{1}^{\delta}$, where $\gamma, \delta \neq 0$ and both have the same sign and sum 1 ; but $\gamma, \delta>0$ and $\gamma+\delta=1$ is impossible. It follows therefore by Proposition 6 that we can write

$$
b_{1} b_{2}=c_{1} \cdot c_{2}, \quad b_{2} b_{1}=d_{1} \cdot d_{2}, \quad c_{2}\left(b_{1} b_{2}\right)^{h}=d_{2}\left(b_{2} b_{1}\right)^{k},
$$

with $h, k \in Z, h, k \geqq 0, c_{1}, d_{1} \neq 1, R\left(c_{1}\right) \neq R\left(d_{1}\right)$.

Comparison of lengths in $F_{0}$ of images of $c_{2}\left(b_{1} b_{2}\right)^{k}$ and $c_{2}\left(b_{2} b_{1}\right)^{k}$ shows that $h=k$; moreover, if $h=k>0$, the terminal segments $\bar{\rho}\left(b_{1} b_{2}\right)$ and $\bar{\rho}\left(b_{2} b_{1}\right)$ of equal length must coincide for all $\rho$, giving $\bar{\rho}\left(b_{1}^{-1} b_{2}^{-1} b_{1} b_{2}\right)=1$, whence, by Proposition $2^{\prime}, b_{1}^{-1} b_{2}^{-1} b_{1} b_{2}=1$, which has been shown impossible. We conclude that $h=k=0$, whence $c_{2}=d_{2}$. If $c_{2}=d_{2}=1$, then $R\left(b_{1} b_{2}\right) \neq R\left(b_{2} b_{1}\right)$ and

$$
a^{h}=\left(b_{1} b_{2}\right)^{\beta}\left(b_{2} b_{1}\right)^{\beta} b_{2}\left(b_{2} b_{1}\right)^{\beta} b_{2}\left(b_{2} b_{1}\right)^{\beta} \cdots \text {, normal. }
$$

Otherwise, the normalizing process gives

$$
\begin{aligned}
a^{2} & =\left(b_{1} b_{2}\right)^{\beta} b_{2}\left(b_{1} b_{2}\right)^{\beta} b_{2}=\left(b_{1} b_{2}\right)^{\beta}\left(b_{2} b_{1}\right)^{\beta} b_{2}^{2} \\
& =\left(b_{1} b_{2}\right)^{\beta-1} c_{1}\left(d_{2} d_{1}\right)^{\beta} b_{2}^{2} \\
& =\left(b_{1} b_{2}\right)^{\beta-1} c_{1}\left(d_{2} d_{1}\right)^{\beta^{\prime}} b^{\prime}, \text { normal, }
\end{aligned}
$$

for some $\beta^{\prime}$ and $b^{\prime}$. Similarly,

$$
a\left(b_{1} b_{2}\right)^{\beta-1} c_{1}=\left(b_{1} b_{2}\right)^{\beta-1} c_{1}\left(d_{2} d_{1}\right)^{\beta \prime \prime} b^{\prime \prime}, \text { normal, }
$$

for some $\beta^{\prime \prime}$ and $b^{\prime \prime}$. It follows that

$$
a^{h}=\left(b_{1} b_{2}\right)^{\beta-1} c_{1}\left(d_{2} d_{1}\right)^{\beta^{\prime \prime}}\left[b^{\prime \prime}\left(d_{2} d_{1}\right)^{\beta^{\prime \prime}}\right]^{h-3} b^{\prime \prime}\left(d_{2} d_{1}\right)^{\beta^{\prime}} b^{\prime},
$$

normal, realizing (1) and (2).

For $l(a)=1$ this establishes that, for some $a_{1}, a_{2}, a_{3}$ and some $d$ in $F_{t}$, for all $h \geqq 4$,

$$
a^{h}=a_{1} a_{2} a_{3}^{h-4} d \text { normal. }
$$


Hence

$$
a a_{1} a_{2} a_{3}^{h-4} d=a a^{h}=a^{h+1}=a_{1} a_{2} a_{3}^{h-3} d,
$$

whence $a a_{1} a_{2}=a_{1} a_{2} a_{3}$, that is $\left(a_{1} a_{2}\right)^{-1} a\left(a_{1} a_{2}\right)=a_{3}$, which establishes (3).

CASE 2. $l(a)=l\left(a^{\prime}\right)>1$. Write $a=b_{1} c_{1}^{\gamma_{1}} \cdots b_{m} c_{m}^{\gamma_{m}} b_{m+1}$ normal, $m>1$. Now

$$
a^{\prime}=b_{2} c_{2}^{\gamma_{2}} \cdots b_{m} c_{m}^{\gamma_{m}} b_{m+1} b_{1} c_{1}^{\gamma_{1}}
$$

is reduced to its normal form

$$
a^{\prime}=d_{2} e_{2}^{\epsilon_{2}} \cdots d_{m} e_{m} d_{m+1} e_{m+1}^{\epsilon_{m+1}} d_{m+2}
$$

by normalizing the part $c_{m}^{\gamma_{m}} b_{m+1} b_{1} c_{1}^{\gamma_{1}}$. The hypothesis that $l(a)=l\left(a^{\prime}\right)$ implies that this part has length 2 , whence from the nature of the normalizing process it follows that $e_{m}=c_{m}$ and that $R\left(e_{m+1} d_{m+2}\right)=R\left(c_{1}\right)$.

We iterate this to find the normal form

$$
a^{\prime \prime}=u_{3} v_{3}^{\alpha_{3}} \cdots u_{m+1} v_{m+1}^{\alpha_{m+1}} u_{m+2} v_{m+2}^{\alpha_{m+2}} w
$$

of $a^{\prime \prime}=d_{3} e_{3}^{\epsilon_{3}} \cdots e_{m+1}^{\epsilon_{m+1}} d_{m+1} d_{2} e_{2}^{\epsilon_{2}}$ by replacing $e_{m+1}^{\epsilon_{m+1}} d_{m+1} d_{2} e_{2}^{\epsilon_{2}}$ by its normal form $v_{m+1}^{\alpha_{m+1}} u_{m+1} v_{m+2}^{\alpha_{m+2}} w$. From $e_{m}=c_{m}$ we infer that, even if $m=2, d_{2}=b_{2}$ and $e_{2}=c_{2}$. From this and the normality of $c_{1}^{\gamma_{1}} b_{2} c_{2}^{\gamma_{2}}$, together with $R\left(e_{m+1} d_{m+2}\right)=R\left(c_{1}\right)$ we conclude that the part in question can fail of normality only by $\mathrm{N} 5$, and the process for establishing N5 shows that $v_{m+1}=e_{m+1}, v_{m+2}=e_{m+2}$, and $w=1$.

If we define $a_{1}=b_{1} c_{1}^{\gamma_{1}}, a_{2}=d_{2} e_{2}^{\epsilon_{2}}$, and $a_{i+m}=a_{i}=u_{i} v_{i}^{\alpha_{i}}$ for $i \geqq 3$, the normality of the products $a_{1} a_{2} a_{3} \cdots a_{k}$ requires only the observation that, since $e_{2}^{k_{2}} d_{3} e_{3}$ was normal, and $v_{m+2}=e_{2}, u_{3}=d_{3}, v_{m+3}=e_{3}$, then $v_{m+2}^{\alpha_{m+2}} u_{3} v_{m+3}^{\alpha_{m+3}}$ is normal as it stands. It is now clear that (1) and (2) hold, and that $a^{\prime \prime}=\left(a_{1} a_{2}\right)^{-1} a\left(a_{1} a_{2}\right)$ $=a_{3} a_{4} \cdots a_{m+2}$, reduced.

CASE 3. $l(a)>l\left(a^{\prime}\right)$. Since $l(a) \geqq l\left(a^{\prime}\right) \geqq l\left(a^{\prime \prime}\right) \geqq \cdots \geqq 1$, clearly for some $k<l(a)$ and $b=a^{(k)}, l(b)=l\left(b^{\prime}\right)$, whence $b$ satisfies (1), (2), (3). Thus $b^{h}$ $=b_{1} b_{2} \ldots$ normal, $b_{i}=w_{i} z_{t}^{\beta_{i}}$, with $b_{i+m}=b_{i}$ for $i>t$ and

$$
c=\left(b_{1} b_{2} \cdots b_{t}\right)^{-1} b\left(b_{1} b_{2} \cdots b_{t}\right)=b_{t+1} b_{t+2} \cdots b_{t+m} .
$$

Replacing $b$ by its cyclic conjugate $c$, we have the same conditions with $t=0$, and $b$ cyclically reduced. Thus, for some $p$ and $q, a=p \cdot q$ and $c=q p$. By Lemma A, $q=q^{\prime} \cdot e, p=e^{-1} \cdot p^{\prime}, c=q^{\prime} \cdot p^{\prime}$. Further, $a=e^{-1} \cdot p^{\prime} \cdot q^{\prime} \cdot e$, reduced, whence $a^{h}=e^{-1} \cdot p^{\prime} \cdot\left(q^{\prime} \cdot p^{\prime}\right)^{h-1} \cdot q^{\prime} \cdot e=e^{-1} \cdot p^{\prime} \cdot b^{h-1} \cdot q^{\prime} \cdot e=e^{-1} \cdot p^{\prime} \cdot b_{1} b_{2} \ldots$, reduced. Since $e^{-1} \cdot p^{\prime} \cdot b_{1} \cdot b_{2}$ is reduced, the normal form of $e^{-1} \cdot p^{\prime} \cdot b_{1} \cdot w_{2}$ ends with the same letters as $b_{1} w_{2}$, whence the normal form $a_{1} a_{2} \cdots a_{n}$ of $e^{-1} \cdot p^{\prime}$ $\cdot b_{1} \cdot b_{2}$ ends in $z_{2}^{\beta_{2}}$, and $a^{h}=a_{1} a_{2} \cdots a_{k} b_{3} b_{4} \cdots$, normal. Thus $a$ satisfies (1) and (2), while for (3) it suffices to note that $d=a_{1} a_{2} \cdots a_{k} b_{3} b_{4} \cdots b_{3 m}$ $=e^{-1} p^{\prime} b^{3}$, whence $d b d^{-1}=e^{-1} p^{\prime} b p^{-1} e=e^{-1} p^{\prime} q p p^{-1} e=e^{-1} p^{\prime} q^{\prime} e=a$, and $b=d^{-1} a d$.

Proof of Proposition $4^{\prime}$. Let $a$ be in $F_{t}$. If some conjugate $p^{-1} a p=b^{\alpha}, b$ in 
$F_{t-1}$, then by Proposition $4, b=c^{\beta}$ for some $c$ primitive, whence $c^{\prime}=p c p^{-1}$ also is primitive and $a=c^{\prime \beta \alpha}$. Otherwise we suppose that $a$ has no cyclic conjugate a power of an element in $F_{t-1}$, whence, by Lemma E, $l(a) \geqq 1$. If $a=b^{\alpha}$ for some $b$ in $F_{t}$, then $b$ satisfies the same condition, and hence (1), (2), and (3) of Lemma $E$. In view of (3), we can replace $b$ by a cyclically reduced conjugate whence, if $b=b_{1} b_{2} \cdots b_{m}$ normal, then $b^{2}=b_{1} b_{2} \cdots b_{m} b_{1} \cdots b_{m}$ normal, and $l\left(b^{2}\right)=2 l(b)$. Now, if $\alpha>1, a=b^{2} \cdot b^{\alpha-2}$. In general, $l(u \cdot v) \geqq l(u)$ $+l(v)-1, l(u \cdot v) \geqq l(u)$. Thus we have $l(a) \geqq l\left(b^{2}\right)=2 l(b)>l(b)$. We conclude first that if $l(a)=1$, then $a=b^{\alpha}$ for $\alpha>1$ is impossible, and $a$ is primitive. Second, if $a$ is not primitive, then $a=b^{\alpha}$ for some $b$ and some $\alpha>1$, and we argue by induction on length that $b=c^{\beta}$ for some primitive $c$, whence $a=c^{\alpha \beta}$, as required.

Proof of Proposition 5'. We show first that if $b$ in $F_{t}$ is cyclically reduced and primitive, and $b$ is not in $F_{t-1}$, then no cyclic conjugate $b^{\prime}$ of $b$ is a power of an element in $F_{t-1}$. For, if $b^{\prime}$ were such a power, since $b^{\prime}$ is primitive, $b^{\prime}$ itself would be in $F_{t-1}$. Then, for some $p$ and $q, b=p \cdot q, b^{\prime}=q p$, and, since $b$ is cyclically reduced, $b^{\prime}=q \cdot p$. From the fact that $b^{\prime}$ is in $F_{t-1}$, that is, $l\left(b^{\prime}\right)=0$, we conclude that $p$ and $q$ are in $F_{t-1}$, whence $b$ is in $F_{t-1}$, contrary to hypothesis.

To prove Proposition $5^{\prime}$, we suppose $a$ in $F_{t}$, and $b$ in $F_{t}$, cyclically reduced, primitive, and not in $F_{t-1}$. It follows from the above and Lemma E that $l\left(b^{h}\right) \geqq h$ for all $h$ in $Z$. Choose $h>l(a)$, whence $l\left(b^{h}\right)>l(a)$. By Lemma A we may write $a=c \cdot d, b^{-h}=d^{-1} \cdot e, a b^{-h}=c \cdot e$. Now $e=1$ would imply $d^{-1}=b^{-h}$, hence $a=c \cdot d=c \cdot b^{h}$ and $l(a) \geqq l\left(b^{h}\right)$, a contradiction. Hence $e \neq 1$. Now choose a new $h \geqq 0$, minimal such that, for some $c, d, e, a=c \cdot d, b^{-h}=d^{-1} \cdot e, a b^{-h}$ $=c \cdot e$, and $e \neq 1$. Since $b^{-h}=d^{-1} \cdot e$ and $e \neq 1$, we conclude that $h>0$. Now $b \cdot b^{h-1}=e^{-1} \cdot d$, and one alternative under Lemma $\mathrm{B}$ is that $e^{-1}=b \cdot f, b^{h-1}$ $=f \cdot d$. Then $b^{-(h-1)}=d^{-1} \cdot f^{-1}$ and, since $a b^{-h}=c \cdot e=c \cdot f^{-1} \cdot b^{-1}, a b^{-(h-1)}=c \cdot f^{-1}$, whence the minimality of $h$ implies that $f=1$. This gives $a=c \cdot d=c \cdot b^{h}$, and, since $c \cdot e=c \cdot b^{-1}, R(c) \neq R(b)$, as required for Proposition $5^{\prime}$. The other alternative is that $b=e^{-1} \cdot f$ and $d=f \cdot b^{h-1}$. In this case we have $a=c \cdot d=c \cdot f \cdot b^{h-1}$, $b=e^{-1} \cdot f, e^{-1} \neq 1$, and, since $c \cdot e, R(c) \neq R\left(e^{-1}\right)$, again satisfying Proposition $5^{\prime}$.

LEMMA $F$. Let $a$ and $b$ be in $F_{b}$, and neither have as cyclic conjugate a power of an element of $F_{t-1}$. Then either $a=c^{h}, b=c^{k}$ for some $c \in F_{t}, h, k \in Z, h, k \geqq 0$, or $a^{-h} b^{h}=a^{-1} \cdot c \cdot b$ for some $c \in F_{t}, h \in Z, h \geqq 0$.

Proof. By Lemma E, paralleling the notation of the lemma, $a^{h}=a_{1} a_{2} \cdots$, $b^{h}=b_{1} b_{2} \cdots$, normal, where, for some integers $t \geqq 0$ and $m, m^{\prime}>0$ we have that $a_{i+m}=a_{i}$ and $b_{i+m^{\prime}}=b_{i}$ for all $i>t$, and also that, writing $p=a_{1} a_{2} \cdots a_{t}$, $q=b_{1} b_{2} \cdots b_{t}, a^{\prime}=p^{-1} a p=a_{t+1} a_{t+2} \cdots a_{t+m}$ and $b^{\prime}=q^{-1} b q=b_{t+1} b_{t+2} \cdots b_{t+m^{\prime}}$. If any first $a_{i} \neq b_{i}$, then Lemma $C$ yields $a_{i+2}^{-1} a_{i+1}^{-1} a_{i}^{-1} b_{i} b_{i+1} b_{i+2}=a_{i+2}^{-1} \cdot c \cdot b_{i+2}$ reduced, hence, for large $h, a^{-h} b^{h}=a^{-1} \cdot c^{\prime} \cdot b$ reduced, as required for Lemma $\mathrm{F}$. If, on the othẹr hand, $a_{i}=b_{i}$ for all $i$, we have $a_{i+n}=a_{i}$ where $n=\left(m, m^{\prime}\right)$, 
and also $p=q$. Writing $d=a_{t+1} a_{t+2} \cdots a_{t+n}$ it follows that $a^{\prime}=d^{m / n}$ and $b^{\prime}=d^{m^{\prime} / n}$, whence $a=c^{h}, b=c^{k}$ where $c=p d p^{-1}, h=m / n$, and $k=m^{\prime} / n$.

Proof of Proposition $6^{\prime}$. We are given $a$ and $b$ in $F_{t}$, cyclically reduced, primitive, and neither in $F_{t-1}$. The reasoning that began the proof of Proposition $5^{\prime}$ shows that both $a$ and $b$ satisfy the hypotheses of Lemma F. If, in accordance with that lemma, $a=c^{h}, b=c^{k}$, then, since $a$ and $b$ are primitive, $a=b^{ \pm 1}$. Either $a=b$, or $a=b^{-1}$ and $a^{-1} b=a^{-1} \cdot 1 \cdot b$ reduced, whence the conclusion of Proposition $6^{\prime}$ holds. Otherwise, by Lemma F, some $a^{-h} b^{k}=a^{-1} \cdot c \cdot b$ and again Proposition $6^{\prime}$ holds.

Proof of Proposition $7{ }^{\prime}$. We are given $a$ and $b$ in $F_{t}$ such that $a b=b a$.

CASE 1. Suppose neither $a$ nor $b$ has as cyclic conjugate a power of an element in $F_{t-1}$. Then the same is true of $a^{-1}$ and $b^{-1}$. Unless $a=c^{h}$ and $b=c^{k}$ for some $c$ in $F_{t}$ and $h, k$ in $Z$, three applications of Lemma $G$ yield an integer $h>0$ such that

$$
a^{h} b^{h}=a \cdot c_{1} \cdot b, \quad b^{h} a^{-h}=b \cdot c_{2} \cdot a^{-1}, \quad a^{-h} b^{-h}=a^{-1} \cdot c_{3} \cdot b^{-1},
$$

all reduced. We note that it follows from Proposition $3^{\prime}$ that, if $a \neq 1, h>0$, then $a^{h}$ begins and ends with $L(a)$ and $R(a)$. It follows that

$$
a^{2 h} b^{2 h} a^{-2 h} b^{-2 h}=a^{h+1} c_{1} b^{2} c_{2} a^{-2} c_{3} b^{-(h+1)}
$$

without cancellation between the seven factors displayed, whence, by the Corollary, $a^{2 h} b^{2 h} a^{-2 h} b^{-2 h} \neq 1, a^{2 h}$ and $b^{2 h}$ do not commute, which contradicts $a b=b a$. This establishes the conclusion in Case 1 .

CASE 2. One of $a$ and $b$, say $a$, is conjugate to a power of an element in $F_{t-1}$. Conjugating $a$ and $b$ simultaneously, we can suppose $a$ itself if a power of an element in $F_{t-1}, a=v^{\alpha}, v$ in $F_{t-1}$, and we can suppose further, apart from the trivial case $a=1$, that $\alpha>0$ and $v$ is cyclically reduced and primitive.

CASE 2A. $\alpha$ in $Z$. Then $a$ is in $F_{t-1}$. If $l(b)=0$, then $b$ is also in $F_{t-1}$ and the conclusion follows by Proposition 7; in view of Proposition 4, in fact, $b=v^{\beta}$ for some $\beta$. Suppose $l(b)=n>0$, and $b=w_{1} z_{1}^{\beta_{1}} \cdots w_{n+1}$ normal. From the normalizing process we see that $b a$ will have a normal form beginning with $w_{1} z_{1}^{\beta}$ for some $\beta$. The normal form of $a b$ will begin with some $r(q p)^{\beta^{\prime}}$ where $z_{1}=p \cdot q, p \neq 1$, and $a w_{1}=r \cdot q \cdot(p \cdot q)^{k}$ reduced, for some $k$ in $Z$. By Lemma D, from $a b=b a$ we conclude that $w_{1}=r$ and $z_{1}=q p$. An argument in the proof of Lemma E shows that $z_{1}=p \cdot q=q \cdot p$ primitive, $p \neq 1$, implies $q=1$. Thus $a w_{1}=r(p q)^{k}=w_{1} z_{1}^{k}$, and $a=w_{1} z_{1}^{k} w_{1}^{-1}$. Since $w_{1} z_{1}$ is normal, if $w_{1} \neq 1$ then $R\left(w_{1}\right) \neq L\left(z_{1}\right)^{-1}$ and $L\left(w_{1}^{-1}\right)=R\left(w_{1}\right)^{-1} \neq R\left(z_{1}\right)^{-1}$, and $a=w_{1} z^{k} w_{1}^{-1}$ is reduced, but not cyclically reduced, contrary to the hypothesis on $a$. Thus $w_{1}=1$, $a=z_{1}^{k}$, whence $a$ commutes with $b^{\prime}=w_{2} z_{2}^{\beta_{2}} \cdots w_{n+1}$. By induction on the length of $b$ we conclude that $b^{\prime}=v^{\beta}$ for some $\beta$, whence $b=v^{\beta+\beta_{1}}$, as required.

CASE 2B. $\alpha$ is not in $Z$. Then $a=v^{\alpha}$ is normal. Again we reason by induction on $l(b)$. The case $l(b)=0$ falls under Case $2 \mathrm{~A}$ with $a$ and $b$ interchanged. Let $b$ have normal form as before, with $l(b)=n>0$. If $w_{1}=1$ and $z_{1}=v$ the 
conclusion follows by induction on $l(b)$ as before. Otherwise, by Lemma C, $v^{\alpha} w_{1} z_{1}^{\beta_{1}}=v^{\alpha^{\prime}} \cdot c \cdot z_{1}^{\beta}$ reduced, and the normal form of $a b$ begins with $v^{\alpha^{\prime \prime}}$ for some $\alpha^{\prime \prime}$. To examine the normal form of $b a$, we observe that the normal form of $w_{n} z_{n}^{\beta_{n}} w_{n+1} v^{\alpha}$ will begin with $w_{n} z_{n}^{\beta}$ for some $\beta^{\prime}$, unless $z_{n}^{\beta_{n}} w_{n+1} v^{\alpha}=z_{n}^{\beta_{n}} q(p q)^{\gamma}$ $=z_{n}^{\beta_{n}}(q p)^{\gamma} q=(q p)^{\delta} q$ where $v=p \cdot q$, and $z^{ \pm 1}=q \cdot p$. In this case, $z_{n}^{\boldsymbol{\beta}_{n}} w_{n+1}$ $=(q p)^{\delta} q(p q)^{-\alpha}=q(p q)^{\epsilon}=q v^{\epsilon}$, and $b=w_{1} q v^{\epsilon}$, whence $a$ commutes with $w_{1} q$ in $F_{t-1}$, and the initial case of the induction gives $w_{1} q=v^{\beta}$, whence $b=v^{\beta+\epsilon}$. Thus, even if $n=1$, the normal form of $b a$ will begin with $w_{1} z_{1}^{\beta^{\prime \prime}}$ for some $\beta^{\prime \prime}$. Since $a b=a b$, this contradicts Lemma D unless $w_{1}=1$ and $z_{1}=v_{1}$.

This completes the proof of Proposition $7^{\prime}$, and with it the proof of Theorem I.

5. Conclusions. Our main result is the following.

THEOREM II. There exists an effective procedure that associates with each expression $\epsilon$, made up out of the symbols $a_{1}, a_{2}, \cdots, a_{r}$ by means of the $X$-group operations, where $X=Z\left[\nu_{1}, \nu_{2}, \cdots, \nu_{d}\right]$, an element $\alpha$ in $X$, in such a way that, if $w$ is the element of the free $X$-group $F$ represented by $\epsilon$, and $\rho$ is any retraction of $X$ onto $Z$, then $\bar{\rho} w=1$ if and only if $\rho \alpha=0$.

Taking into account the $X$-group axioms, we can replace $\epsilon$ by an expression $\epsilon^{\prime}$ representing the same group element $w$, where $\epsilon^{\prime}=\epsilon_{1}^{\alpha_{1}} \epsilon_{2}^{\alpha_{2}} \cdots \epsilon_{n}^{\alpha_{n}}, n \geqq 0$, all $\alpha_{i}$ in $X$, and where each $\epsilon_{i}$ represents an element $w_{i}$ of $F_{t}$, for some fixed $t \geqq 0$. Let $\omega=w_{1}^{\alpha_{1}} w_{2}^{\alpha_{2}} \cdots w_{n}^{\alpha_{n}}$, and let $C$ be the empty set of conditions. Then $(\omega, C)$ is a word of height $t+1$, and, by Theorem $\mathrm{I},(\omega, C)$ is equivalent to a finite set of normal words $\left(\omega_{1}, C_{1}\right),\left(\omega_{2}, C_{2}\right), \cdots,\left(\omega_{k}, C_{k}\right)$. We may suppose these words indexed in such a way that, for some $h, 0 \leqq h \leqq k, \omega_{1}=1, \omega_{2}$ $=1, \cdots, \omega_{h}=1$, while none of $\omega_{h+1}, \omega_{h+2}, \cdots, \omega_{k}$ is the empty word. Since every retraction $\rho$ satisfies the vacuous set of conditions $C$, it follows from the definition of equivalence that every retraction satisfies one of $C_{1}, C_{2}, \cdots, C_{k}$. If $\rho$ satisfies some $C_{i}$ for $1 \leqq i \leqq h$, then $\bar{\rho} w=\bar{\rho} 1=1$; while if $\rho$ satisfies some $C_{i}$ for $i>h$, then $\bar{\rho} w=\bar{\rho} w_{i}$, where $w_{i}$ is the element represented by $\omega_{i}$, and from conditions N2 and N3 for the normality of $\omega_{i}$, it follows that $\bar{p} w_{i}$ has a left letter and a right letter, and hence $\bar{\rho} w w_{i} \neq 1$.

For each $i$, after transposing, we can write the finite set of equations in $C_{i}$ in the form $\rho \alpha_{i 1}=0, \rho \alpha_{i 2}=0, \cdots, \rho \alpha_{i m_{i}}=0, m_{i} \geqq 0$. Define $\alpha_{i}=\alpha_{i 1}^{2}+\alpha_{i 2}^{2}$ $+\cdots+\alpha_{i m_{i}}^{2}$, and set $\alpha=\alpha_{1} \alpha_{2} \cdots \alpha_{h}$. If $\bar{\rho} w=1$, then $\rho$ satisfies some $C_{i}$, for $1 \leqq i \leqq h$, hence $\rho \alpha_{i j}=0$ for $j=1,2, \cdots, m_{i}, \rho \alpha_{i}=0$, and thus $\rho \alpha=0$. Suppose conversely that $\rho \alpha=0$. Then $h \geqq 1$, and $\rho \alpha_{i}=0$ for some $i, 1 \leqq i \leqq h$, whence $\rho \alpha_{i j}=0$ for $j=1,2, \cdots, m_{i}$. Now $\left(\omega, C_{i}\right)$ is equivalent to $\left(1, C_{i}\right)$, by virtue of (E2) and (E3) alone, which make no reference to any inequalities contained in $C_{i}$; that is, $\omega$ can be transformed into 1 by means of the $X$-group axioms together with substitutions implied by the equations $\alpha_{i 1}=0, \alpha_{i 2}$ $=0, \cdots, \alpha_{l m_{i}}=0$. Since $\rho \alpha_{i j}=0$ for $j=1,2, \cdots, m_{i}$, it follows that $\bar{\rho} w$ 
$=\bar{\rho} 1=1$. Thus we have shown that, for every retraction $\rho, \bar{\rho} w=1$ if and only if $\rho \alpha=0$, which completes the proof to the theorem.

We conclude by justifying an assertion made in the introduction that, for $X=Z\left[\nu_{1}, \nu_{2}, \cdots, \nu_{d}\right], d \geqq 0$, the axioms given for $X$-groups are neither too strong nor too weak in the sense that an expression $\epsilon$ representing an element of the free $X$-group $F$ reduces to 1 by virtue of these axioms if and only if, under every substitution of integers for $\nu_{1}, \nu_{2}, \cdots, \nu_{d}, \epsilon$ is transformed into an ordinary word representing the element 1 of the ordinary free group $F_{0}$.

It is clear that the axioms are not too strong: if they permit us to transform one expression $\epsilon$ into a second $\epsilon^{\prime}$, surely $\epsilon$ and $\epsilon^{\prime}$ represent the same element of $F_{0}$ under each substitution. To show that the axioms are strong enough we must show that if $\epsilon$ represents an element $w$ of $F$ such that $\bar{\rho} w=1$ under all retractions $\rho$, then in fact $w=1$, the trivial element of $F$. Returning to the notation used in the proof of Theorem II, we have that $\rho \alpha=0$ for all $\rho$. This implies that $\alpha=0$, identically in $X$, whence $h \geqq 1$ and $\alpha_{i}=0$ for some $i, 1 \leqq i \leqq h$. But $\alpha_{i}=\alpha_{i 1}^{2}+\alpha_{i 2}^{2}+\cdots+\alpha_{i m_{i}}^{2}=0$ implies that $\alpha_{i j}=0$ for all $j=1,2, \cdots, m_{i}$, so that $C_{i}$ in fact contains no nontrivial conditions. But now, as before, $\omega$ reduces to 1 by means of the axioms together with the trivial condition $0=0$, that is, by means of the axioms alone.

\section{BIBLIOGRAPHY}

1. R. Baer, Abelian groups without elements of finite order, Duke Math. J. vol. 3 (1937) p. 68.

2. G. Baumslag, Some aspects of groups with unique roots, Acta Math. vol. 103 (1960). 1957.

3. P. Hall, Nilpotent groups, Canadian Mathematical Congress, University of Alberta,

4. P. G. Kontorovich, On the theory of noncommutative torsion free groups, Doklady Akad. Nauk SSSR. vol. 59 (1948) p. 213.

5. M. Lazard, Sur les groupes nilpotents et les anneaux de Lie, Ann. Sci. Ecole Norm. Sup. (3) vol. 71 (1954) p. 101.

6. A. I. Malcev, Nilpotent torsion free groups, Izv. Akad. Nauk SSSR. Ser. Mat. vol. 13 (1949) p. 201.

7. B. H. Neumann, Adjunction of elements to groups, J. London Math. Soc. vol. 18 (1943) p. 12 .

8. V. A. Tartakovski, Application of the sieve method to the solution of the word problem for certain types of groups, Mat. Sb. N.S. vol. 25 (67) (1949) p. 251.

UNIVERSITY OF MiCHIGAN, Ann Arbor, Michigan

Institute for Defense ANALyses, Princeton, New Jersey 